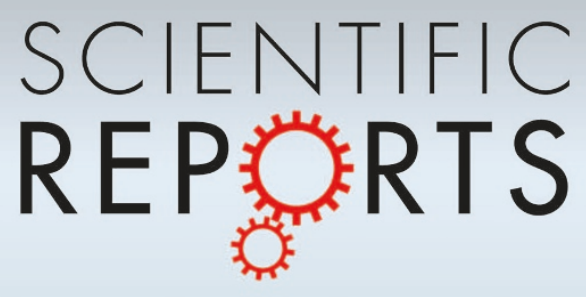

\title{
OPEN \\ SUBJECT AREAS: \\ CHEMICAL BIOLOGY \\ A chemical genomic study identifying diversity in cell migration signaling in cancer cells
}

CANCER

COLLECTIVE CELL MIGRATION

MESENCHYMAL MIGRATION

Received

22 August 2012

Accepted

17 October 2012

Published

8 November 2012

Correspondence and requests for materials should be addressed to M.l. (imoto@bio.keio. ac.jp)

\author{
Shigeyuki Magi, Etsu Tashiro \& Masaya Imoto
}

Department of Biosciences and Informatics, Faculty of Science and Technology, Keio University, 3-14-1 Hiyoshi, Kohoku-ku, Yokohama 223-8522, Japan.

The aim of this study was to analyze the diversity and consistency of regulatory signaling in cancer cell migration, using a chemical genomic approach. The effects of 34 small molecular compounds were assessed quantitatively by wound healing assay in ten types of migrating cells. Hierarchical clustering was performed on the subsequent migration inhibition profile of the compounds and cancer cell types. The result was that hierarchical clustering accurately classified the compounds according to their targets. Furthermore, the cancer cells tested in this study were classified into three clusters, and the compounds were grouped into four clusters. An inhibitor of JNK suppressed all types of cell migration; however, inhibitors of ROCK, GSK-3 and p38MAPK only inhibited the migration of a subset of cell lines. Thus, our analytical system could easily distinguish between the common and cell type-specific signals responsible for cell migration.

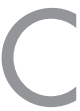

ell migration is central to many physiological processes, including development, tissue remodeling, and immune responses, and is also a required step in cancer metastasis. When a cell moves, multiple intracellular signaling networks control cell morphology. Signaling can be initiated through receptor tyrosine kinases, G protein-coupled receptors (GPCRs), integrin, and other receptors. These receptors are upregulated by extracellular stimuli that induce the activation of one or more intermediate signaling network branches. Finally, this signaling reaches the Rho family of small GTPase proteins. Many molecules and pathways have been implicated in intermediate signaling. For example, the Ras/Raf/MEK/ERK pathway has been reported to enhance cell motility ${ }^{1-4}$. In addition to the Ras/Raf/MEK/ERK pathway, a phosphoinositide 3-OH kinase (PI3K)/Akt pathway is widely known to regulate cell migration. This pathway is considered to be necessary for both Cdc42and Rac1-induced cell motility and invasiveness ${ }^{5}$, and it regulates the expression of Snail, which can increase cell motility ${ }^{6}$. Jun NH2-terminal kinase (JNK) and p38 mitogen-activated protein kinase (p38MAPK) have also been reported to play important roles in the signaling mechanisms involved in migration ${ }^{7,8}$. The role of Rho family small GTPase proteins, which is considered to constitute the final stage of the migration-signaling network, is known to regulate actin nucleation and polymerization. In particular, RhoA, Racl, and Cdc42 are the major regulators of cytoskeletal remodeling. Activation of RhoA increases cell contractility and leads to the formation of focal adhesions and stress fibers'. Rac1 and Cdc42 activation induce the lamellipodia and filopodia, respectively ${ }^{10,11}$. Thus, the core elements of the intracellular migration-signaling network have been demonstrated.

However, it is likely that signaling molecules regulating cell migration in one cancer cell may not regulate cell migration in other genetically distinct cancer cells. Indeed, the PI3K/Akt pathway, but not the MEK/ERK pathway, has been shown to be critical for prostate cancer cell migration ${ }^{6}$. Other studies have reported that the constitutive activation of the MEK/ERK pathway by oncogenic mutations of BRaf ${ }^{\mathrm{V} 600 \mathrm{E}}$ significantly induced cell migration through activation of RhoA GTPase ${ }^{12}$. In addition, the role of the Rho family of proteins in cell migration depends on specific cellular circumstances. The migration of several types of cancer cell is based on reorganization of the actin cytoskeleton, but their requirements for Rho and Rac signaling differ. With respect to a particular subset of cancer cells, cells migrated in a Rac-dependent manner, but Rho signaling was not essential. With respect to another subset of cancer cells, the inhibition of Rho/Rock signaling inhibited cell migration. Thus, although the same basic process of cell migration is induced, each type of cancer cell brings about migration in different contexts using distinct molecular repertoires. Therefore, understanding the diversity and commonality of signaling pathways that regulate cell migration in various cell types is important not only for basic research into cell migration, but also for the development of anti-metastatic anti-tumor drugs. 
Table 1 | The experimental conditions of the wound healing assays for each cell line

\begin{tabular}{llcc} 
Cell line & \multicolumn{1}{c}{ Origin } & Cell number (cells/well) & Migration factor \\
\hline A431 & Human epithelial carcinoma & $7.5 \times 10^{4}$ & EGF 3 $\mathrm{ng} / \mathrm{ml}$ or EC17-CM \\
EC17 & Human esophageal carcinoma & $7.5 \times 10^{4}$ & $\mathrm{None}$ \\
EC109 & Human esophageal carcinoma & $7.5 \times 10^{4}$ & $\mathrm{EGF} 3 \mathrm{ng} / \mathrm{ml}$ or EC17-CM \\
HT1080 & Human fibrosarcoma & $7.5 \times 10^{4}$ & $\mathrm{FBS}(2 \%)$ \\
TE8 & Human esophageal carcinoma & $7.5 \times 10^{4}$ & $\mathrm{EGF} 3 \mathrm{ng} / \mathrm{ml}$ \\
T1 & Human medullary thyroid carcinoma & $7.5 \times 10^{4}$ & $\mathrm{EGF} 3 \mathrm{ng} / \mathrm{ml}$ \\
3Y1 & Rat fibroblast & $7.5 \times 10^{4}$ & $\mathrm{FBS}(5 \%)$ \\
B16 & Mouse melanoma & $2.2 \times 10^{5}$ & $\mathrm{EC} 17-\mathrm{CM}$ \\
\hline
\end{tabular}

To address this issue, we utilized the chemical genomic approach in which chemical inhibitors were used as probes to mimic loss-offunction phenotypes by inhibiting target protein activity; that is, if a chemical inhibitor suppresses the cell migration of one type of cancer cell, the target protein of the inhibitor can be considered as being involved in the mechanism of cell migration of that type of cell. This chemical genetic approach is easily applicable to different cell models; therefore, it can determine which signaling molecule is universally involved in the migration mechanism in several types of cancer cells, and which one is specifically involved in each type of cell. In the present study, we first examined the effects of various chemical inhibitors on cell migration in several cancer cell models, and subsequently obtained chemosensitive migratory profiles and undertook cluster analysis to classify the signaling molecules and their inhibitors as being either common to all cancer cells or specific to certain cell types.

\section{Results}

Determination of appropriate experimental conditions for the wound healing assay. To select the cell models used in this study, sixteen cell lines, including colon carcinoma, esophageal carcinoma and lung cancer, were assessed with regard to their migration ability in response to migration factors using a wound healing assay ${ }^{13}$. The assay conditions of each cell line were optimized by examining migration factors such as growth factors, cell number required to maintain a confluent cell monolayer, and an assay duration that clearly revealed the extent of motility. We found out that the eight cell lines were suitable for use in a migration assay under the conditions indicated in Table 1 (see also the Methods section). We also confirmed that both number of alive and dead cells in each condition were not clearly increased in optimized assay condition. The other cell lines tested were not affected by migratory stimuli or could not be scratched. Among the eight cell lines selected, EC17 cells migrated without extracellular stimulation, indicating that EC17 cells secrete chemoattractants into the media, and acquire motility by autocrine signaling. Conversely, others required the addition of migration factors, such as epidermal growth factor (EGF), conditioned medium from EC17 cells (EC17-CM), or serum (Figure 1a). A431 cells and EC109 cells migrated in response to both EGF and EC17-CM. Figure 1b shows the morphology of migration in these cell lines. A431 cells and EC109 cells moved together in sheet-like structures (collective migration), whereas the other cell lines showed a fibroblast-like spindle-shaped morphology and migrated individually like mesenchymal cells (mesenchymal migration).

Signaling pathway regulating for cell migration differs among three cancer cell lines. Next, to examine whether our analytical system could distinguish between common signals responsible for cell migration in the cancer cells tested and cell type-specific signals, using signal transduction inhibitors, a test was done using A431 cells, EC109 cells or TT cells that were randomly selected to analyze their migration ability. This was conducted following treatment with three a

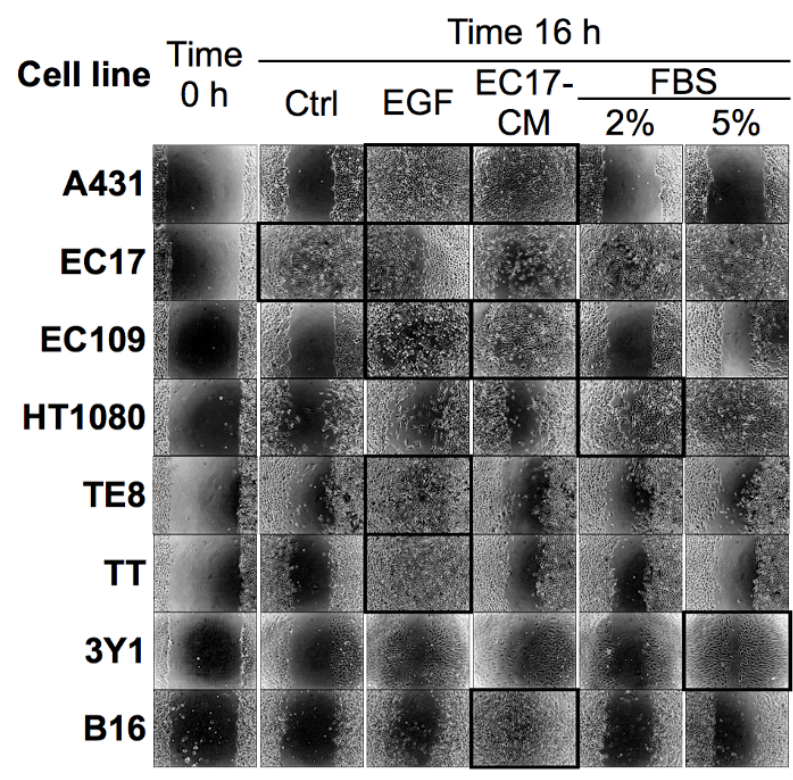

b

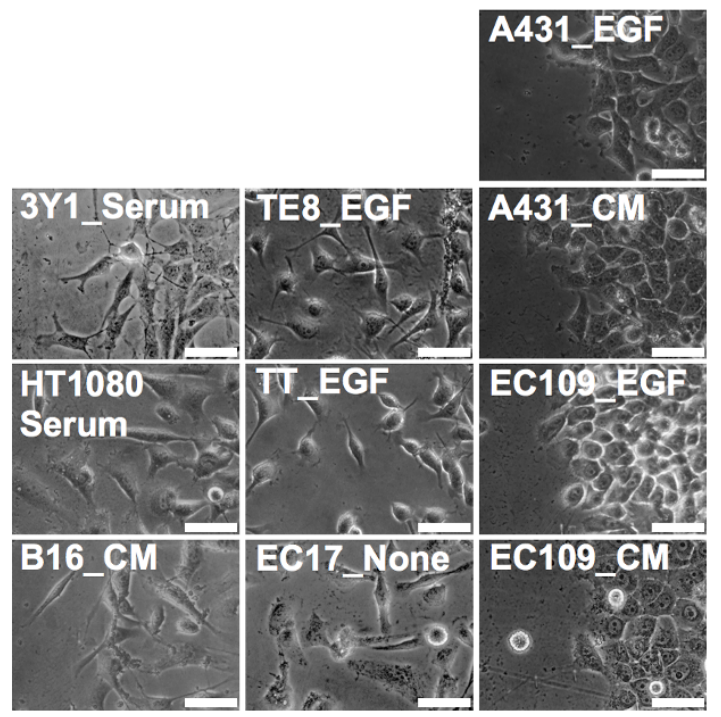

Figure 1 The effect of migratory stimuli on cell migration in various cell lines. (a) Cells were scratched and then stimulated by EGF $(3 \mathrm{ng} / \mathrm{mL})$, serum, or conditioned medium from EC17 cells. After $16 \mathrm{~h}$, wound areas were observed and photographed under microscopy. (b) Images of cell lines treated with migratory stimuli. Cells were photographed $10 \mathrm{~h}$ after stimulation. The scale bar represents $50 \mu \mathrm{m}$. 


\begin{tabular}{|c|c|c|c|c|c|c|c|c|c|}
\hline \multirow[t]{3}{*}{ Ctrl } & \multicolumn{9}{|c|}{ EGF $3 \mathrm{ng} / \mathrm{mL}$} \\
\hline & \multicolumn{3}{|c|}{ AG1478 $(\mu \mathrm{M})$} & \multicolumn{3}{|c|}{ LY294002 (нM) } & \multicolumn{3}{|c|}{ Y27632 (uM) } \\
\hline & 0.1 & 0.3 & 1 & 3 & 10 & 30 & 10 & 30 & 100 \\
\hline
\end{tabular}
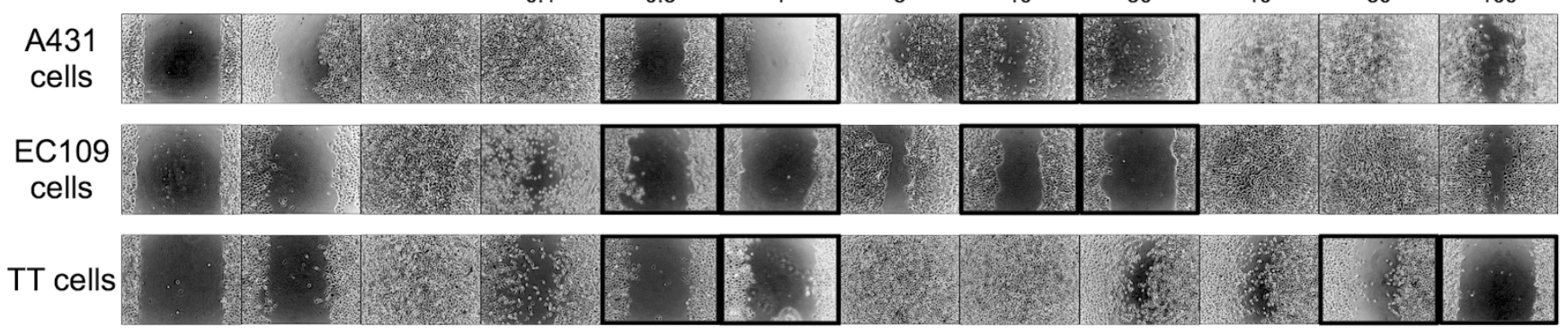

b

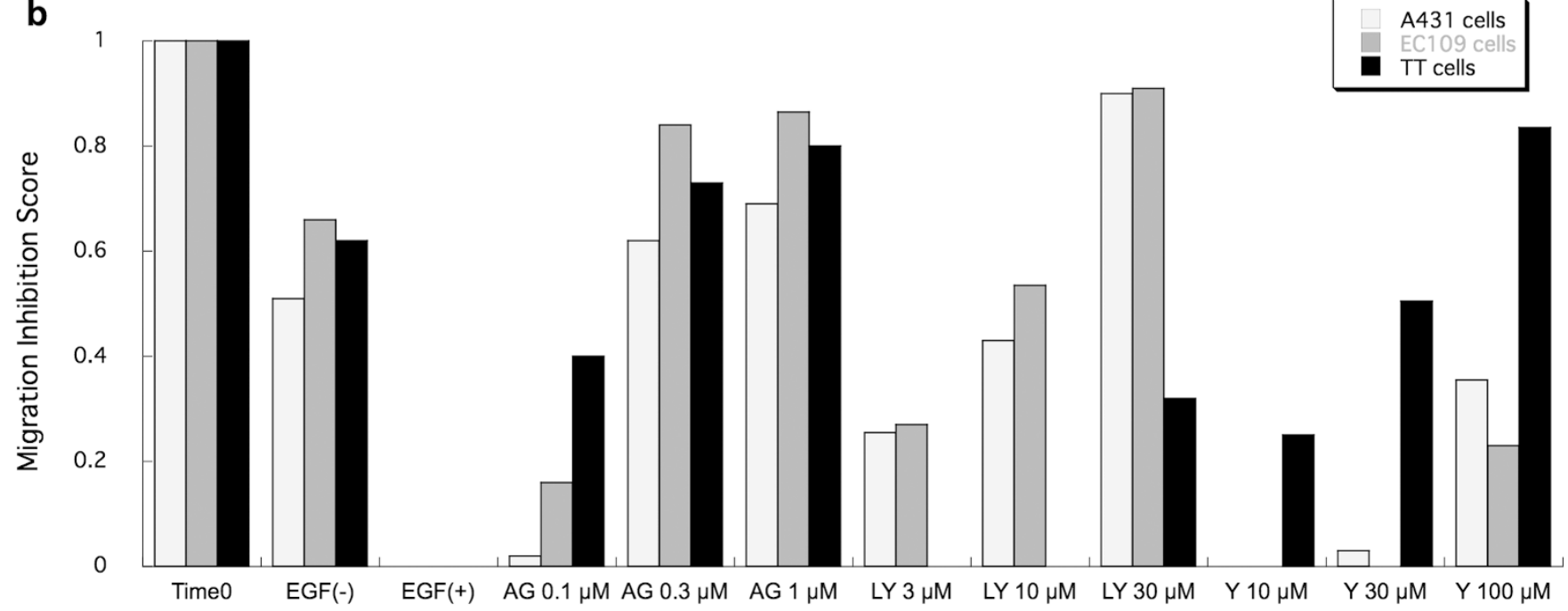

Figure 2 The inhibitory pattern of cell migration was dependent on the types of cancer cell line. A confluent monolayer of A431 cells, EC109 cells, and TT cells were scratched, treated with AG1478, LY294002, or Y27632, and stimulated with EGF as described in the Methods section. (a) Wound areas were photographed just after scratching (time zero). After $16 \mathrm{~h}$, wound areas were photographed again (others). Black boxes indicate the inhibitory effects of chemicals on cell migration. The data were representative of two independent studies. (b) Migration inhibition score (MIS) of each experimental condition. MIS was quantified by measurement of the cell-free area in the picture. The quantified value was normalized against the value at time zero. The data were the average of two independent studies.

kinase inhibitors; PI3K inhibitor, Rho-associated kinase (ROCK) inhibitor and EGF receptor kinase inhibitor. The reason why we focused on the inhibitors of PI3K and ROCK for this test was that $\mathrm{PI} 3 \mathrm{~K}$ and ROCK were expected to reveal cell type-specific effects on migration. This is because they have been reported to be involved in regulation mechanisms of cell migration that are initiated downstream to growth factor signaling in a subset of cancer cells $s^{5,14}$, although they were also reported to be dispensable for migration or membrane ruffling in certain conditions ${ }^{15,16}$. Figure 2a presents the effect of these three inhibitors on the EGF-induced motility of A431 cells, EC109 cells, and TT cells. The extent of the cell motility was quantified by the measurement of the cell-free area in a photograph. The quantified value was calculated over a fixed period of time, and was termed the 'migration inhibition score (MIS)' (Figure 2b). These results indicated that the EGF receptor kinase inhibitor, AG1478, inhibited the EGF-induced migration of all three cell lines, as expected. The PI3K inhibitor and LY294002 suppressed the EGF-induced migration of A431 cells and EC109 cells, but not of TT cells, indicating that PI3K plays a critical role in EGFinduced cell migration in A431 cells and EC109 cells. In contrast, the ROCK inhibitor, Y27632, suppressed migration only in A431 cells and TT cells, indicating that ROCK is indispensable for EGF-induced cell migration in A431 cells and TT cells but not in EC109 cells. Thus, our analytical system using chemical inhibitors of signal transduction easily distinguished between common and cell typespecific signals responsible for cell migration.

Two-way cluster analysis of migration inhibition score. To reveal the diversity and generality of regulatory signaling in cancer cell migration, we tested the effect of 34 different signal transduction inhibitors on the migration of ten types of cells, as shown in Table 1. Table 2 lists the names of the chemical inhibitors of signal transduction used in this study, the experimental concentrations of each inhibitor, and their modes of action. Each inhibitor was used at three concentrations, the highest one being a concentration just below the level that would affect cell viability. Using these chemical inhibitors under the stated concentrations, we carried out two highly reproducible, independent experiments on each cell line $(r=0.94, p-$ value $<2.2 \times 10^{-16}$, Figure 3 ), and provided a final dataset by averaging the data points from the two experiments. The MIS dataset is shown in Supplementary Table 1. Then a hierarchical cluster analysis was performed. The results are displayed in the form of a heat map and a tree diagram (Figure 4). The heat map employs a gradient color scale from green, indicating MIS $=0$, to magenta, indicating MIS $=1.0$, interpolated over black indicating MIS $=0.5$.

As a result of these experiments, the characteristic features of cell migration affected by chemical inhibitors in cancer cells were 
Table 2 | Compound concentrations and targets of inhibition used in this study

\begin{tabular}{|c|c|c|c|}
\hline Compound name & Concentration & Target / Mode of action & References \\
\hline A23187 & $30,100,300 \mathrm{nM}$ & $\mathrm{Ca}^{2+}$ lonophore & 33 \\
\hline AA861 & $3,10,30 \mu \mathrm{M}$ & 5-Lipoxygenase & 34 \\
\hline AG 1478 & $0.1,0.3,1 \mu \mathrm{M}$ & EGFR & 35 \\
\hline ALLN & $1,3,10 \mu \mathrm{M}$ & Calpain & 37 \\
\hline Bafilomycin A & $0.3,1,3 \mathrm{nM}$ & V-ATPase & 38 \\
\hline Cytochalasin D & $0.1,0.3,1 \mu \mathrm{M}$ & Actin polymarization & 39 \\
\hline LY294002 & $3,10,30 \mu \mathrm{M}$ & $\mathrm{PI} 3 \mathrm{~K}$ & 42 \\
\hline Mevastatin & $3,10,30 \mu \mathrm{M}$ & HMG-CoA reductase & 43 \\
\hline Moverastin & $3,10,30 \mu \mathrm{M}$ & Farnesyl transferase & 20 \\
\hline MG132 & $10,30,100 \mathrm{nM}$ & Proteasome & 44 \\
\hline MK571 & $3,10,30 \mu \mathrm{M}$ & CysLT 1 & 45 \\
\hline MK886 & $1,3,10 \mu \mathrm{M}$ & FLAP & 46 \\
\hline Risedronate & $30,100,300 \mu \mathrm{M}$ & FPP synthase & 36 \\
\hline SB203580 & $3,10,30 \mu \mathrm{M}$ & p38MAPK & 52 \\
\hline SB2 18078 & $30,100,300 \mathrm{nM}$ & Chk 1 & 53 \\
\hline SB4 15286 & $3,10,30 \mu \mathrm{M}$ & GSK-3 & 54 \\
\hline SP600125 & $1,3,10 \mu \mathrm{M}$ & JNK & 55 \\
\hline Thapsigargin & $3,10,30 \mathrm{nM}$ & $\mathrm{Ca}^{2+}$-ATPase & 56 \\
\hline Trichostatin A & $30,100,300 \mathrm{ng} / \mathrm{mL}$ & Histone deacetylase & 57 \\
\hline Tunicamycin & $30,100,300 \mathrm{ng} / \mathrm{mL}$ & Glycosylation & 58 \\
\hline U0126 & $3,10,30 \mu \mathrm{M}$ & MEK & 59 \\
\hline UTKOI & $1,3,10 \mu \mathrm{M}$ & $14-3-3$ & 22 \\
\hline Vinblastine & $3,10,30 \mathrm{ng} / \mathrm{mL}$ & Tubulin polymerization & 60 \\
\hline Wortmannin & $0.3,1,3 \mu \mathrm{M}$ & PI3K & 61 \\
\hline Xanthohumol & $0.3,1,3 \mu \mathrm{g} / \mathrm{mL}$ & Valosin-containing protein & 23 \\
\hline
\end{tabular}

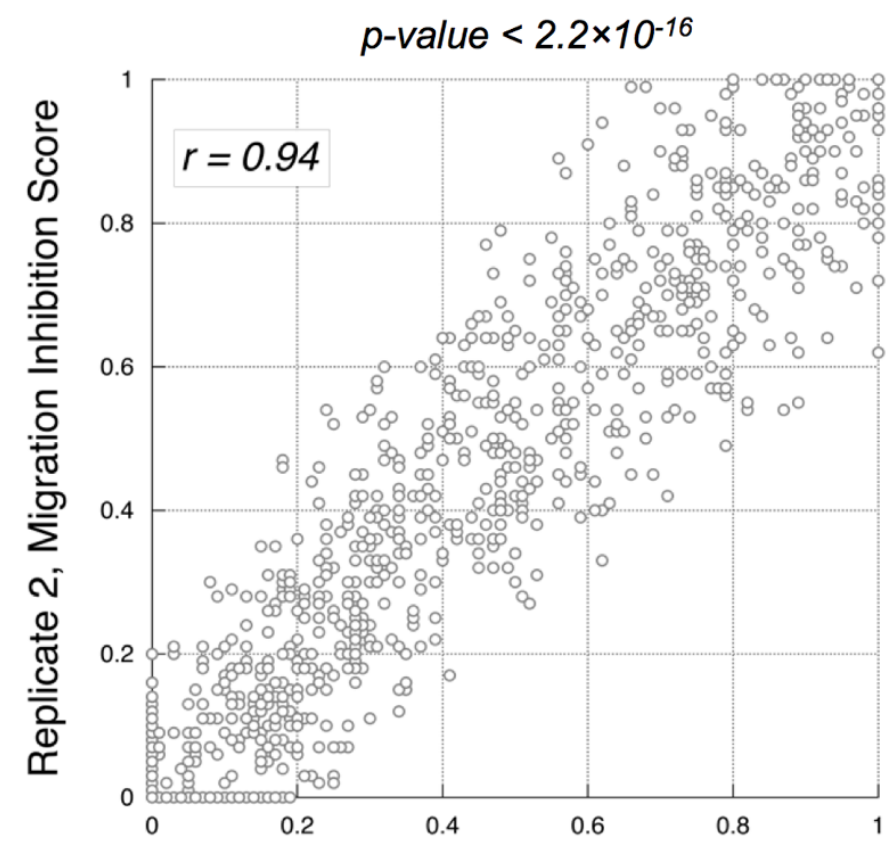

Replicate 1, Migration Inhibition Score

Figure 3 Reproducibility of the migration inhibition score. Before averaging, two independent data sets were checked for high correlation $\left(\mathrm{r}=0.94, \mathrm{p}\right.$-value $\left.<2.2 \times 10^{-16}\right)$. classified into three general clusters (Figure 4a). Cluster A consisted of three types of cells and cell migration properties: B16 cells, HT1080 cells, and 3Y1 cells; their cell migration displayed lower sensitivities to the inhibitors tested in this study than the others (Figure 4b). Cluster B consisted of cell migration of A431 cells and EC109 cells stimulated with either EGF or EC17-CM. The EGFinduced chemosensitive migratory profile of these cells was similar to that induced by EC17-CM. Cluster C consisted of three types of cells: EC17 cells, TE8 cells and TT cells.

It was expected that the chemical inhibitors that targeted the same molecule would be clustered into the same tree. Indeed, PD169316 and SB203580 as p38MAPK inhibitors, herbimycin A and radicicol (Hsp90 inhibitors), LY294002 and wortmannin (PI3K inhibitors), paclitaxel and vinblastine (tubulin binders), and alendronate and risedronate (farnesyl diphosphate (FPP) synthase inhibitors), were all clustered into the same position (indicated by gray boxes). These results indicate that our chemical genomic approach was able to classify the chemical inhibitors based on their respective modes of action, similar to previous studies on the chemosensitivities of cancer cells ${ }^{17-19}$.

Furthermore, the chemical inhibitors used in this study were classified into four general clusters (Figure $4 b$ ), and each inhibitor in Figure $4 \mathrm{~b}$ can be linked to its target molecule. We also displayed the relationships of the targets of the inhibitors as a non-root phylogenetic tree (Figure 4c). The inhibitors grouped into cluster 1 contained the 5-lipoxygenase-activating protein (FLAP) inhibitor, MK886, the vacuolar-type proton-ATPase (V-ATPase) inhibitor, bafilomycin A, and the FPP synthase inhibitors, the bisphosphonates. These inhibitors showed little inhibitory effect on cell migration in almost all cell types, thus the target molecules of these compounds had little bearing 
a
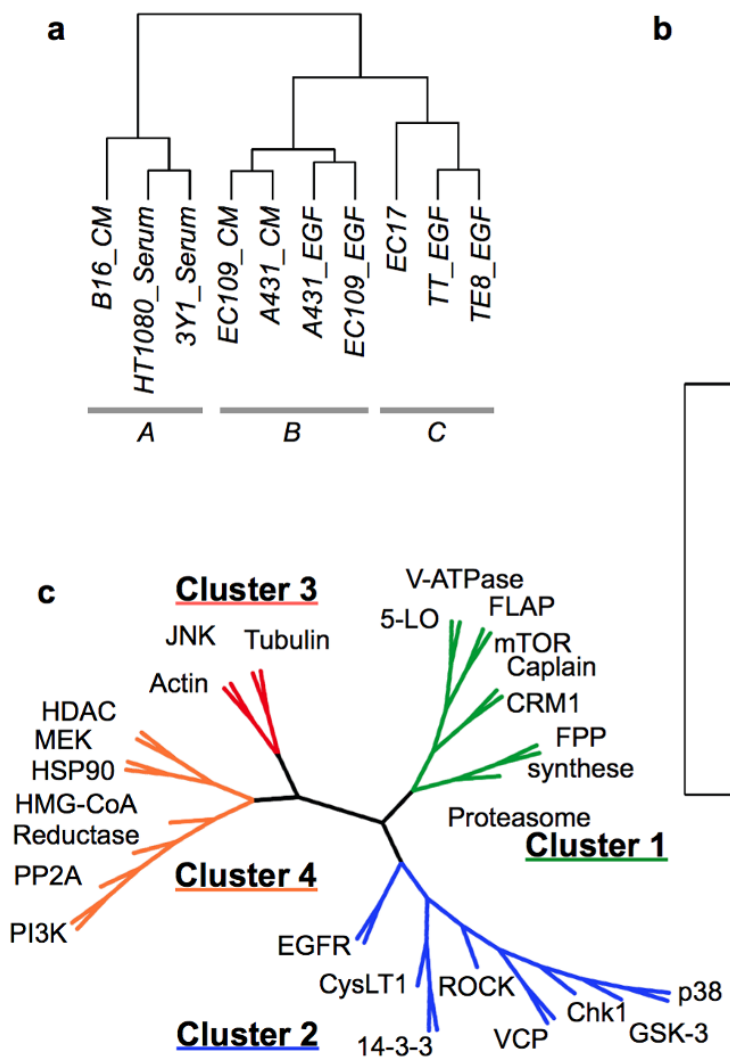

b

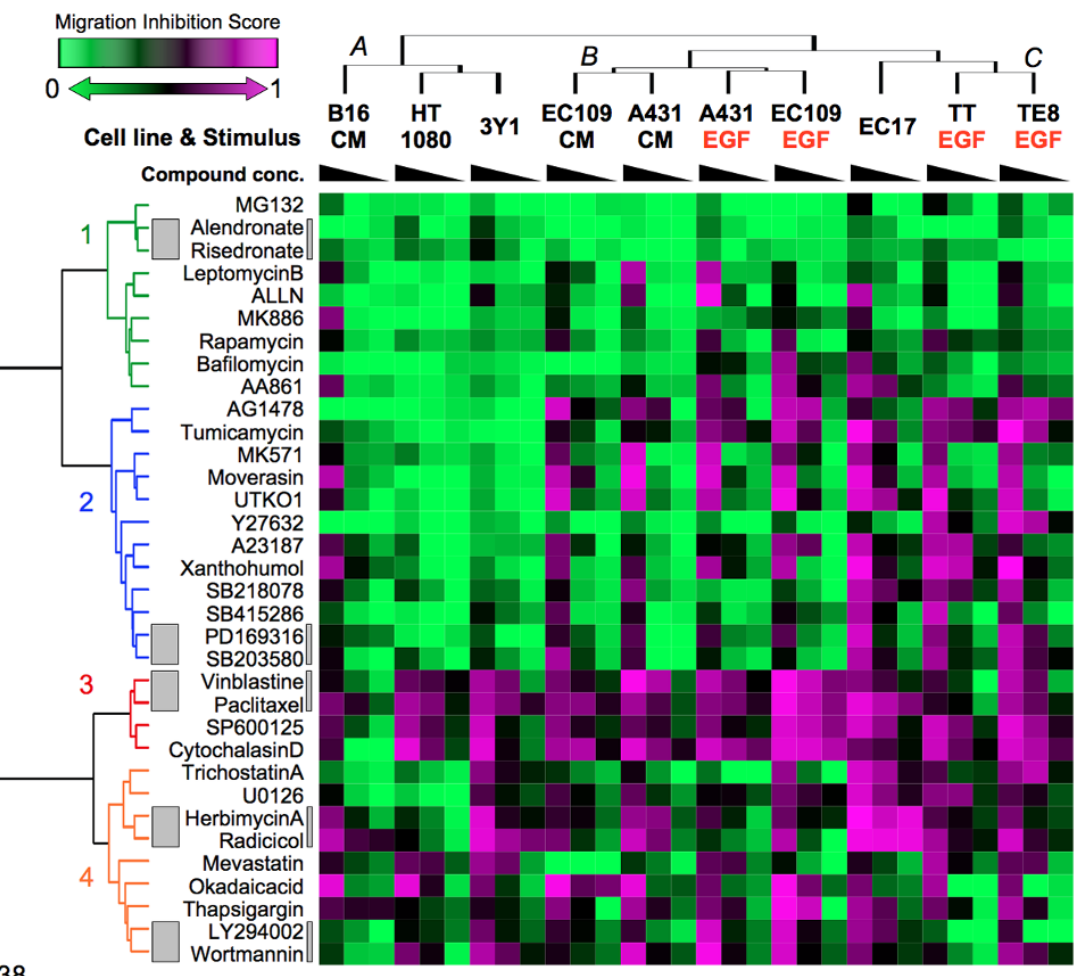

Figure 4 | Cluster analysis of the chemosensitivity profile of migration inhibition. Cluster analysis was performed using Euclidean distance and Ward's method. (a) The MIS dataset was clustered into ten types of cell migration. Cell migration types were classified into three general clusters; clusters A, B, and C. (b) The MIS dataset was hierarchically clustered using data from 34 compounds. Rows indicate 34 different small molecular compounds. Columns indicate the ten migration types, including the three different compound concentrations. The heat map shows a gradient color scale from green, indicating MIS $=0$, to magenta, indicating MIS $=1$, interpolated over black for MIS $=0.5$. Gray boxes beside the heat map indicate that two labeled compounds have almost the same molecular target. The 34 compounds were clustered into four general groups. (c) The non-rooted phylogenetic tree classifies the target molecules of the small molecular compounds tested in this study. Each small compound inhibitor used in this study can be replaced with its target molecule because most targets have already been identified. This phylogenetic tree presents the distances between molecules on the signaling network contributing to cell migration.

on the regulating mechanisms of cell migration tested in this study. Cluster 2 contained Y27632, AG1478, the p38MAPK inhibitors, the chk1 inhibitor, SB218078 and so on. Most of these compounds showed a stronger inhibitory effect on cell migration, classified into migration type clusters B and C, in contrast to cluster A. Therefore, the target molecules of these compounds were not involved in the migration of HT1080 cells and 3Y1 cells but they did regulate cell migration in the subset of cell lines grouped into clusters B and C. Cluster 3 contained SP600125 and the cytoskeleton-affecting compounds. This group of inhibitors affected all types of cell migration, indicating that not only cytoskeletal molecules, but also JNK, are common regulators of cell migration, irrespective of cell type. Cluster 4 contains the Hsp90 inhibitors, the MEK inhibitor, U0126, and the PI3K inhibitors. These inhibitors also suppressed migration in all types of cell with different potencies depending on cell type. Thus the target molecules of these inhibitors also played a common role in all types of cell migration.

\section{Discussion}

In the present study, we investigated some general and specific regulatory mechanisms of cell migration. To accomplish our objective, we assessed the effects of 34 different kinds of chemical inhibitors on the migration of ten types of cells using a wound healing assay, and subsequently performed a cluster analysis on the dataset. One significant aspect of this work is that each compound showed a characteristic cell type-specific inhibitory pattern on migration, and hierarchical clustering precisely classified the compounds according to their respective targets, such as p38, Hsp90, PI3K, tubulin and FPP synthase (Figure $4 \mathrm{~b}, \mathrm{c}$ ). Therefore, our research could be applied to predict the mode of action of each compound. For example, moverastin and its derivative UTKO1 were classified into same cluster, in spite of the different functions of these two compounds, which have similar structures. We previously reported that moverastin was an inhibitor of farnesyltransferase $e^{20}$, and it inhibited cell migration by inhibiting farnesylation in H-Ras. On the other hand, UTKO1 was reported not to inhibit farnesyltransferase $e^{21}$, but it directly bound to 14-3-3 , and inhibited the interaction between the 14-3-3 proteins and Tiam1, a protein that has been reported to be a Rac-specific GEF. This resulted in the inhibition of $\mathrm{Racl}^{22}$. Therefore, it has been demonstrated that the migration of epithelial cells requires Taim1mediated Rac1 activation. However, because our profiling data demonstrated that moverastin and UTKO1 were classified in the same cluster, we examined the possibility that moverastin could bind to 14-3-3 , and obtained similar results (data not shown). Therefore it is likely that moverastin might inhibit cell migration not only by inhibiting protein farnesylation, but also by inhibiting Tiam1mediated Rac1 activation. In addition, because the CysLT1 antagonist MK571 was classified into the same cluster as moverastin and UTKO1, this raised the possibility that CysLT1 signaling might be closely related to Taim1-mediated Racl activation. We therefore examined this potential mode of action, and found that EGF-induced Rac1 activation was regulated by CysLT1 signaling (unpublished 
data). Thus, our profiling data appeared to be helpful in the mechanistic study of cell migration. At the same time, our clustering data indicated that xanthohumol was grouped in the same category as the $\mathrm{Ca}^{2+}$ ionophore, A23187. Recently, xanthohumol was reported to bind to and inhibit valosin-containing protein $(\mathrm{VCP})^{23}$, resulting in the induction of ER stress. Although A23187 is also a well-known inducer of ER stress ${ }^{24,25}$, other ER stress-inducing compounds such as tunicamycin and thapsigargin were classified into different clusters from xanthohumol and A23817. Therefore, an interpretation of the clustering data must be made with great caution.

The characteristics of cell migration based on chemical inhibitorsensitivity profiles were grouped into three clusters (clusters A, B and $\mathrm{C}$ ), and chemical inhibitors were classified into four general groups (clusters 1 to 4, Figure 4). Although the motilities of several cell lines we tested are upregulated by extracellular stimuli such as EGF, they migrated a little without stimulation (Figure 1a). Additionally, EC17 cells did not require extracellular stimulation to migrate. Thus we just have to evaluate and discuss chemical inhibitor-sensitivity profiles as total effects on "basal" and "stimulated" motility. JNK inhibitor, tubulin and actin polymerization inhibitors in cluster 3 showed a potent inhibitory effect on migration of all cell types, indicating that JNK is a common and crucial signaling molecule regulating cell migration. Indeed, JNK was reported to modulate migration in a broad range of cell types ${ }^{26}$, such as keratinocytes ${ }^{7}$, neuronal cells ${ }^{27}$, and many cancer cell lines ${ }^{28,29}$. Because dynamic reorganization of the actin cytoskeleton is considered to be key to the cell's capacity to migrate $^{30}$, JNK may be indispensable for the phosphorylation of paxillin and F-actin polymerization ${ }^{7,8}$.

In contrast, some of the chemical inhibitors classified into cluster 2 (AG1478, tunicamycin, the CysLT1 antagonist MK571, Moverastin and UTKO1) affected the migration of cell lines of epithelial origin (EC109 cells, A431 cells, EC17 cells, TT cells, TE8 cells) in clusters B and C, but did not affect the migration of cell lines of mesenchymal origin (HT1080 cells and 3Y1 cells) placed in cluster A. This suggests that there is an essentially different regulatory mechanism of cell migration between cells of these two origins. The regulatory mechanism of cell migration of B16 cells appeared somewhat similar to that of cell lines of mesenchymal origin when compared to those with an epithelial origin.

Moreover, although EGF did induce migration of cells of epithelial origin (TT cells, TE8 cells, A431 and EC109 cells), differential sensitivities to several inhibitors was observed in A431 and EC109 cells in cluster B and TT cells and TE8 cells in cluster $\mathrm{C}$. These results suggest that the EGF signaling pathway leading to migration of A431 and EC109 cells in cluster B was not identical to that of TT cells and TE8 cells in cluster C. Furthermore, EC17-CM also induced migration of A431 and EC109 cells with a similar chemosensitive profile to that seen in EGF-induced migration, indicating that EC17-CM might activate almost the same signaling pathway as the EGF-signaling pathway in the context of the migration of these cells. One exception is AA861, an inhibitor of 5-lipoxygenase (5-LO). AA861 inhibited the EGF-induced migration of A431 cells and EC109 cells, but not EC17-CM-induced migration. Therefore, production of leukotriene(s) catalyzed by 5-lipoxygenase is required for EGF-induced cell migration, whereas EC17-CM may already contain leukotriene(s), so EC17-CM-induced cell migration was not inhibited by inhibition of 5-LO. In addition, a specific inhibitor of EGF-receptor tyrosine kinase (AG1478) potently inhibited the EC17-CM-induced migration of A431 and EC109 cells, but weakly inhibited migration of EC17 cells, indicating that EC17 cells might produce and secrete EGF, whereas EC17 cells underwent cell migration in response to migration factors other than EGF.

Interestingly, the mode of EGF-induced cell migration based on cell morphology can also be classified into clusters B and C. As shown in Figure 1b, A431 cells and EC109 cells in cluster B showed collective migration, whereas TT, TE8 and EC17 cells in cluster C showed a mesenchymal migration. With respect to collective migration, cells moved in groups and a leading cell at the tip of the group generated the migratory traction and the cells in the middle and at the back of the group were predominantly dragged passively. In contrast, mesenchymal migration required the formation of protrusions at the leading edge and actomyosin-mediated retraction of the trailing edge. This raises the possibility that the difference in the mode of cell migration of epithelial cells might be correlated with the differences in sensitivity to chemical inhibitors between clusters B and C. The ROCK inhibitor, Y27632, is a representative example; it inhibited mesenchymal migration of TT cells and TE8 cells more potently than collective migration of A431 and EC109 cells. Indeed, Rho-ROCK signaling is proposed to induce actomyosin-mediated retraction at the trailing edge in mesenchymal migration ${ }^{15}$. As an exception, the ROCK inhibitor Y27632 failed to inhibit mesenchymal migration of EC17 cells. At present, although we do not know why inhibition of ROCK did not suppress the migration of EC17 cells, one possible explanation is that another Rho effector, citron kinase or mDia, could regulate mesenchymal migration if used instead of ROCK. Moreover, the GSK-3 inhibitor (SB415286) and the p38MAPK inhibitors (PD169316 and SB203580) also inhibited the EGF-induced migration of TE8, TT, and EC17 cells more potently than they did in A431 and EC109 cells. This indicates that GSK3 and p38MAPK might be involved in the Rho-ROCK signaling responsible for mesenchymal migration. These ideas can be supported by other findings. GSK-3 phosphorylated and inactivated p190A RhoGAP, which is a key Rho regulatory protein in the context of cell migration. This resulted in the activation of Rho-ROCK signaling ${ }^{31}$. Furthermore, the phosphorylation of protein substrates by GSK-3 often requires the "priming" of a neighboring residue by a distinct kinase, leading to subsequent phosphorylation by GSK- $3^{32}$. p38MAPK could effectively prime the C-terminal fragment of p190A RhoGAP for subsequent phosphorylation by GSK-3. The Chk1 inhibitor SB218078 also inhibited EGF-induced migration of TE8, TT, and EC17 cells more potently than it did in A431 and EC109 cells. However, at present we do not know how chk1 is involved in mesenchymal migration. Moreover, we cannot exclude the possibility that chk1 is important in mechanisms other than the mode of cell migration. Contrastingly, although PI3K, PP2A and HMG-CoA reductase somewhat selectively inhibited EGF-induced collective migration, the role of these enzymes on cell migration remains unclear.

In summary, we have shown that JNK is a signaling molecule common to all types of cell migration, and many molecules have diverse functions in the migration of particular types of cancer cells. We determined this using a chemical genomic approach. Our approach can be used as a tool for understanding the diversity and similarities in cancer cell migration signaling, opening up the potential for revealing novel molecular targets in cancer therapy.

\section{Methods}

Cell culture. 3Y1 and HT1080 cells were maintained in Dulbecco's Eagle's medium (DMEM) supplemented with 10\% fetal bovine serum (FBS), $0.1 \mathrm{~g} / \mathrm{l}$ kanamycin, 100 units/ml penicillin G, $0.6 \mathrm{~g} / \mathrm{l} \mathrm{L-glutamine,} \mathrm{and} 2.5 \mathrm{~g} / 1 \mathrm{NaHCO}_{3}$. A431 cells were maintained in DMEM supplemented with $5 \%$ calf serum (CS), $0.1 \mathrm{~g} / \mathrm{l}$ kanamycin, 100 units/ml penicillin G, $0.6 \mathrm{~g} / \mathrm{l} \mathrm{L}$-glutamine, and $2.5 \mathrm{~g} / 1 \mathrm{NaHCO}_{3}$. B16 cells were maintained in DMEM supplemented with 8\% FBS, $0.1 \mathrm{~g} / \mathrm{l}$ kanamycin, 100 units $/ \mathrm{ml}$ penicillin $\mathrm{G}, 0.6 \mathrm{~g} / \mathrm{l} \mathrm{L}$-glutamine, and $2.5 \mathrm{~g} / \mathrm{NaHCO}_{3}$. EC17, EC109, TE8, and TT cells were maintained in Roswell Park Memorial Institute (RPMI) medium 1640 supplemented with 5\% FBS, $0.1 \mathrm{~g} / \mathrm{l}$ kanamycin, 100 units/ml penicillin G, $0.6 \mathrm{~g} / \mathrm{l} \mathrm{L}$ glutamine, and $2.5 \mathrm{~g} / \mathrm{NaHCO}_{3}$. For routine culture, cells were incubated in a standard humidified incubator at $37^{\circ} \mathrm{C}$ in $5 \% \mathrm{CO}_{2}$.

Reagents. A23187, AG1478, ALLN, cytochalasin D, okadaic acid, rapamycin, and Y27632 were purchased from Calbiochem. MK571 was purchased from Cayman. Thapsigargin was purchased from Santa Cruz Biotechnology. AA861, bafilomycin A, LY294002, mevastatin, MG132, MK886, PD169316, SB203580, SB218078, SB415286, SP600125, tunicamycin, U0126, wortmannin, and epidermal growth factor were purchased from Sigma. Paclitaxel, radicicol, and vinblastine were purchased from Wako Pure Chemical Industries, Ltd. Herbimycin A, moverastin, and xanthohumol was purified from cultures of Streptomyces sp. in our own laboratory. Leptomycin B 
and trichostatin A were kind gifts from Dr. Minoru Yoshida at RIKEN. Compound UTKO1 was synthesized and kindly donated by Dr. Hidenori Watanabe of the University of Tokyo. Alendronate and risedronate were kind gifts from Yamanouchi Pharmaceutical Co., Ltd (Astellas Pharma Inc.).

Preparation of conditioned medium $(\mathrm{CM})$ from EC17 cells. EC17 cells $\left(1.0 \times 10^{6}\right.$ cells) were seeded in $\phi 100 \mathrm{~mm}$ dish. The following day, the medium was replaced with $10 \mathrm{ml}$ of RPMI 1640 containing $1 \%$ FBS. After $24 \mathrm{~h}$, the medium was recovered and sterilized by filtration.

Wound healing assay. A confluent monolayer of cells in a 48-well plate was scratched with a micropipette tip to create a cell-free zone in each well, about $1 \mathrm{~mm}$ in width. The medium was replaced with RPMI1640 with $1 \%$ FBS with or without test compound, and cells were either treated with the migratory stimulus or not treated with it so as to serve as controls. After a fixed period of time, cells were observed and photographed under a microscope. The experimental conditions for each cell line are described as Table 1. Wound areas were quantified using ImageJ software. After photographing, cells were trypsinized and collected, and cell viability was determined by trypan blue dye exclusion assay. Average migration inhibition scores were calculated from two independent experiments.

Cluster analysis. The value of migration inhibition was ordered according to the experimental conditions of cell migration or the compounds used. These profiles were analyzed by hierarchical clustering (using the method of Ward's linkage based on Euclidean distance) and visualized using a heat map using the R Project package (http://www.R-project.org)

1. Marshall, C. J. Ras effectors. Curr. Opin. Cell Biol. 8, 197-204 (1996).

2. Klemke, R. L. et al. Regulation of cell motility by mitogen-activated protein kinase. J. Cell Biol. 137, 481-492 (1997).

3. Nguyen, D. H. et al. Myosin light chain kinase functions downstream of Ras/ERK to promote migration of urokinase-type plasminogen activator-stimulated cells in an integrin-selective manner. J. Cell Biol. 146, 149-164 (1999).

4. Tanimura, S. et al. Prolonged nuclear retention of activated extracellular signalregulated kinase $1 / 2$ is required for hepatocyte growth factor-induced cell motility. J. Biol. Chem. 277, 28256-28264 (2002).

5. Keely, P. J., Westwick, J. K., Whitehead, I. P., Der, C. J. \& Parise, L. V. Cdc42 and Racl induce integrin-mediated cell motility and invasiveness through PI(3)K. Nature 390, 632-636 (1997)

6. Gan, Y. et al. Differential roles of ERK and Akt pathways in regulation of EGFRmediated signaling and motility in prostate cancer cells. Oncogene 29, 4947-4958 (2010)

7. Huang, C., Rajfur, Z., Borchers, C., Schaller, M. D. \& Jacobson, K. JNK phosphorylates paxillin and regulates cell migration. Nature 424, 219-223 (2003).

8. Wagner, E. F. \& Nebreda, A. R. Signal integration by JNK and p38 MAPK pathways in cancer development. Nat. Rev. Cancer 9, 537-549 (2009).

9. Narumiya, S., Ishizaki, T. \& Watanabe, N. Rho effectors and reorganization of actin cytoskeleton. FEBS Lett. 410, 68-72 (1997).

10. Nobes, C. D. \& Hall, A. Rho, rac, and cdc42 GTPases regulate the assembly of multimolecular focal complexes associated with actin stress fibers, lamellipodia, and filopodia. Cell 81, 53-62 (1995).

11. Etienne-Manneville, S. \& Hall, A. Rho GTPases in cell biology. Nature 420, 629-635 (2002).

12. Makrodouli, E. et al. BRAF and RAS oncogenes regulate Rho GTPase pathways to mediate migration and invasion properties in human colon cancer cells: a comparative study. Mol. Cancer 10, 118 (2011).

13. Yarrow, J. C., Totsukawa, G., Charras, G. T. \& Mitchison, T. J. Screening for cell migration inhibitors via automated microscopy reveals a Rho-kinase inhibitor. Chem. Biol. 12, 385-395 (2005).

14. Kakinuma, N., Roy, B. C., Zhu, Y., Wang, Y. \& Kiyama, R. Kank regulates Rho A-dependent formation of actin stress fibers and cell migration via 14-3-3 in PI3K-Akt signaling. J. Cell Biol. 181, 537-549 (2008).

15. Sanz-Moreno, V. et al. Rac activation and inactivation control plasticity of tumor cell movement. Cell 135, 510-523 (2008).

16. Kurokawa, K. et al. Coactivation of Rac1 and Cdc42 at lamellipodia and membrane ruffles induced by epidermal growth factor. Mol. Biol. Cell 15, 1003-1010 (2004).

17. Scherf, U. et al. A gene expression database for the molecular pharmacology of cancer. Nat. Genet. 24, 236-244 (2000)

18. Nakatsu, N. et al. Chemosensitivity profile of cancer cell lines and identification of genes determining chemosensitivity by an integrated bioinformatical approach using cDNA arrays. Mol. Cancer Ther. 4, 399-412 (2005)

19. Muroi, M. et al. Application of proteomic profiling based on 2D-DIGE for classification of compounds according to the mechanism of action. Chem. Biol. 17, 460-470 (2010).

20. Takemoto, Y. et al. Chemistry and biology of moverastins, inhibitors of cancer cell migration, produced by Aspergillus. Chem. Biol. 12, 1337-1347 (2005).

21. Sawada, M. et al. Synthesis and anti-migrative evaluation of moverastin derivatives. Bioorg. Med. Chem. Lett. 21, 1385-1389 (2011).
22. Kobayashi, H. et al. Involvement of 14-3-3 proteins in the second epidermal growth factor-induced wave of Racl activation in the process of cell migration. J. Biol. Chem. 286, 39259-39268 (2011).

23. Sasazawa, Y. et al. Xanthohumol impairs autophagosome maturation through direct inhibition of valosin-containing protein. ACS Chem. Biol. (2012). doi:10.1021/cb200492h

24. Werno, C., Zhou, J. \& Brüne, B. A23187, ionomycin and thapsigargin upregulate mRNA of HIF-1alpha via endoplasmic reticulum stress rather than a rise in intracellular calcium. J. Cell Physiol. 215, 708-714 (2008).

25. Yamazaki, M., Chiba, K. \& Yoshikawa, C. Genipin suppresses A23187-induced cytotoxicity in neuro2a cells. Biol. Pharm. Bull. 32, 1043-1046 (2009).

26. Huang, C., Jacobson, K. \& Schaller, M. D. A role for JNK-paxillin signaling in cell migration. Cell Cycle 3, 4-6 (2004).

27. Sun, Y., Yang, T. \& Xu, Z. The JNK pathway and neuronal migration. J. Genet. Genomics 34, 957-965 (2007).

28. Ching, Y. P. et al. P21-activated protein kinase is overexpressed in hepatocellular carcinoma and enhances cancer metastasis involving c-Jun NH2-terminal kinase activation and paxillin phosphorylation. Cancer Res. 67, 3601-3608 (2007).

29. Wang, J. et al. Sustained c-Jun-NH2-kinase activity promotes epithelialmesenchymal transition, invasion, and survival of breast cancer cells by regulating extracellular signal-regulated kinase activation. Mol. Cancer Res. 8, 266-277 (2010)

30. Pollard, T. D. \& Borisy, G. G. Cellular motility driven by assembly and disassembly of actin filaments. Cell 112, 453-465 (2003).

31. Jiang, W. et al. p190A RhoGAP is a glycogen synthase kinase-3-beta substrate required for polarized cell migration. J. Biol. Chem. 283, 20978-20988 (2008)

32. Harwood, A. J. Signal transduction in development: holding the key. Dev. Cell. 2 , 384-385 (2002)

33. Abbott, B. J. et al. Microbial transformation of A23187, a divalent cation ionophore antibiotic. Antimicrob. Agents Chemother. 16, 808-812 (1979).

34. Yoshimoto, T. et al. 2,3,5-Trimethyl-6-(12-hydroxy-5,10-dodecadiynyl)-1,4benzoquinone (AA861), a selective inhibitor of the 5-lipoxygenase reaction and the biosynthesis of slow-reacting substance of anaphylaxis. Biochim. Biophys. Acta. 713, 470-473 (1982).

35. Osherov, N. \& Levitzki, A. Epidermal-growth-factor-dependent activation of the src-family kinases. Eur. J. Biochem. 225, 1047-1053 (1994).

36. van Beek, E., Pieterman, E., Cohen, L., Löwik, C. \& Papapoulos, S. Farnesyl pyrophosphate synthase is the molecular target of nitrogen-containing bisphosphonates. Biochem. Biophys. Res. Commun. 264, 108-111 (1999).

37. Inoue, S., Bar-Nun, S., Roitelman, J. \& Simoni, R. D. Inhibition of degradation of 3-hydroxy-3-methylglutaryl-coenzyme A reductase in vivo by cysteine protease inhibitors. J. Biol. Chem. 266, 13311-13317 (1991).

38. Bowman, E. J., Siebers, A. \& Altendorf, K. Bafilomycins: a class of inhibitors of membrane ATPases from microorganisms, animal cells, and plant cells. Proc Natl. Acad. Sci. USA 85, 7972-7976 (1988).

39. Cooper, J. A. Effects of cytochalasin and phalloidin on actin. J. Cell Biol. 105, 1473-1478 (1987).

40. Whitesell, L., Mimnaugh, E. G., De Costa, B., Myers, C. E. \& Neckers, L. M Inhibition of heat shock protein HSP90-pp60v-src heteroprotein complex formation by benzoquinone ansamycins: essential role for stress proteins in oncogenic transformation. Proc. Natl. Acad. Sci. USA 91, 8324-8328 (1994).

41. Nishi, K. et al. Leptomycin B targets a regulatory cascade of $\mathrm{crm} 1$, a fission yeast nuclear protein, involved in control of higher order chromosome structure and gene expression. J. Biol. Chem. 269, 6320-6324 (1994).

42. Vlahos, C. J., Matter, W. F., Hui, K. Y. \& Brown, R. F. A specific inhibitor of phosphatidylinositol 3-kinase, 2-(4-morpholinyl)-8-phenyl-4H-1-benzopyran4-one (LY294002). J. Biol. Chem. 269, 5241-5248 (1994).

43. Endo, A., Kuroda, M. \& Tanzawa, K. Competitive inhibition of 3-hydroxy-3methylglutaryl coenzyme A reductase by ML-236A and ML-236B fungal metabolites, having hypocholesterolemic activity. FEBS Lett. 72, 323-326 (1976).

44. Lee, D. H. \& Goldberg, A. L. Proteasome inhibitors: valuable new tools for cell biologists. Trends Cell Biol. 8, 397-403 (1998).

45. Woszczek, G. et al. Functional characterization of human cysteinyl leukotriene 1 receptor gene structure. J. Immunol. 175, 5152-5159 (2005)

46. Ford-Hutchinson, A. W. FLAP: a novel drug target for inhibiting the synthesis of leukotrienes. Trends Pharmacol. Sci. 12, 68-70 (1991).

47. Bialojan, C. \& Takai, A. Inhibitory effect of a marine-sponge toxin, okadaic acid, on protein phosphatases. Specificity and kinetics. Biochem. J. 256, 283-290 (1988).

48. Schiff, P. B. \& Horwitz, S. B. Taxol stabilizes microtubules in mouse fibroblast cells. Proc. Natl. Acad. Sci. USA 77, 1561-1565 (1980).

49. Kummer, J. L., Rao, P. K. \& Heidenreich, K. A. Apoptosis induced by withdrawal of trophic factors is mediated by p38 mitogen-activated protein kinase. J. Biol. Chem. 272, 20490-20494 (1997).

50. Schulte, T. W. et al. Antibiotic radicicol binds to the N-terminal domain of Hsp90 and shares important biologic activities with geldanamycin. Cell Stress Chaperones 3, 100-108 (1998).

51. Brown, E. J. et al. A mammalian protein targeted by G1-arresting rapamycinreceptor complex. Nature 369, 756-758 (1994).

52. Gould, G. W., Cuenda, A., Thomson, F. J. \& Cohen, P. The activation of distinct mitogen-activated protein kinase cascades is required for the stimulation of 
2-deoxyglucose uptake by interleukin-1 and insulin-like growth factor-1 in KB cells. Biochem. J. 311 (Pt 3), 735-738 (1995).

53. Zhao, B. et al. Structural basis for Chk1 inhibition by UCN-01. J. Biol. Chem. 277, 46609-46615 (2002).

54. Coghlan, M. P. et al. Selective small molecule inhibitors of glycogen synthase kinase-3 modulate glycogen metabolism and gene transcription. Chem. Biol. 7, 793-803 (2000).

55. Han, Z. et al. c-Jun $\mathrm{N}$-terminal kinase is required for metalloproteinase expression and joint destruction in inflammatory arthritis. J. Clin. Invest. 108, 73-81 (2001).

56. Thastrup, O., Foder, B. \& Scharff, O. The calcium mobilizing tumor promoting agent, thapsigargin elevates the platelet cytoplasmic free calcium concentration to a higher steady state level. A possible mechanism of action for the tumor promotion. Biochem. Biophys. Res. Commun. 142, 654-660 (1987).

57. Yoshida, M., Kijima, M., Akita, M. \& Beppu, T. Potent and specific inhibition of mammalian histone deacetylase both in vivo and in vitro by trichostatin A. J. Biol. Chem. 265, 17174-17179 (1990).

58. Takatsuki, A., Arima, K. \& Tamura, G. Tunicamycin, a new antibiotic. I. Isolation and characterization of tunicamycin. J. Antibiot. 24, 215-223 (1971).

59. DeSilva, D. R. et al. Inhibition of mitogen-activated protein kinase kinase blocks $T$ cell proliferation but does not induce or prevent anergy. J. Immunol. 160, 4175-4181 (1998).

60. Owellen, R. J., Owens, A. H. \& Donigian, D. W. The binding of vincristine, vinblastine and colchicine to tubulin. Biochem. Biophys. Res. Commun. 47, 685-691 (1972).

61. Arcaro, A. \& Wymann, M. P. Wortmannin is a potent phosphatidylinositol 3 kinase inhibitor: the role of phosphatidylinositol 3,4,5-trisphosphate in neutrophil responses. Biochem. J. 296 (Pt 2), 297-301 (1993).
62. Uehata, M. et al. Calcium sensitization of smooth muscle mediated by a Rhoassociated protein kinase in hypertension. Nature 389, 990-994 (1997).

\section{Acknowledgements}

We would like to thank Dr. M. Yoshida (RIKEN) for his kind gift of leptomycin B and trichostatin A; Dr. H. Watanabe (University of Tokyo) for his gracious gift of UTKO1; Dr. A. Funahashi (Keio University) for kind advice regarding data analysis. This work was supported by a Grant-in-Aid for Challenging Exploratory Research and JSPS Fellows, the Ministry of Education, Culture, Sports, Science, and Technology, Japan.

\section{Author contributions}

M.I. is responsible for project planning and experimental design; S.M. performed all of the experiments and statistical analyses; S.M., E.T. and M.I. co-wrote the paper.

\section{Additional information}

Competing financial interests: The authors declare no competing financial interests.

License: This work is licensed under a Creative Commons

Attribution-NonCommercial-NoDerivs 3.0 Unported License. To view a copy of this license, visit http://creativecommons.org/licenses/by-nc-nd/3.0/

How to cite this article: Magi, S., Tashiro, E. \& Imoto, M. A chemical genomic study identifying diversity in cell migration signaling in cancer cells. Sci. Rep. 2, 823; DOI:10.1038/ srep00823 (2012). 